\title{
Month of birth is not a definite risk factor for multiple sclerosis
}

\author{
0 mês de nascimento não é um fator de risco definitivo para esclerose múltipla
}

The article of Fragoso et al. ${ }^{1}$, recently published in Arquivos de Neuro-Psiquiatria, demonstrating that the month of birth (MOB) does not interfere with the prevalence or progression of multiple sclerosis (MS), adds very important information in this controversial matter. The results obtained in that study, involving 1207 patients and 1207 controls from 4 South America countries, covering a wide latitudinal range, nicely fit the findings of our group, which have also been recently published ${ }^{2}$.

In our study, we compared the MOB of all MS patients born in the northwestern region of Portugal, located between latitudes $41^{\circ} 08^{\prime}$ and 41 $7^{\prime}$, from 1992 to 1943 $(n=421)$ with the MOB found in the control sample composed of the live births records $(n=1,150,362)$ in the same time period and geographical area ${ }^{2}$. As the Chi-square methods have several limitations for seasonality assessment and have been found to be insensitive to seasonal fluctuations of moderate amplitude, we applied the Hewitt test of seasonality $^{3}$, and did not find any significant differences in the MOB of MS patients and controls.

Thus, our data does not support the seasonality hypothesis of "MOB effect" for MS risk in Portugal, which is opposite to the widely divulged statement among the MS scientific community, whereby in the Northern hemisphere those born in winter have a reduced MS risk and those born in spring have an increased risk. Despite this statement has been based in the most cited large-scale study conducted in the Northern hemisphere ${ }^{4}$, recently emphasized in a systematic review and meta-analysis ${ }^{5}$, the truth is that no significant differences in the MOB of MS patients comparing with controls have been observed in some populations with high MS prevalence, as Canada/British Columbia ${ }^{2}$, or still significance varied with the statistical test ${ }^{2}$, suggesting that the association between birth season and risk of MS might simply be a statistical artifact.

In conclusion, the absence of a seasonality pattern concerning the MOB of MS patients from Latin America and Portugal shows that MOB is not a definite risk factor for MS. This finding casts doubt about the real meaning of the assumption that those born in springtime in the Northern hemisphere countries have a higher risk for MS, which has rapidly spread among MS experts. In addition, studies with findings opposite to the established version of "MOB effect in MS" must be taken into account in further meta-analysis with real worldwide coverage.

Maria José Sá

\section{References}

1. Fragoso YD, Adoni T, Almeida SM, et al. Multiple sclerosis in South America: month of birth in different latitudes does not seem to interfere with the prevalence or progression of the disease. Arq Neuropsiquiatr 2013;71:573-579.

2. Barros P, Marques de Sá J, Sá MJ. Month of birth and risk of multiple sclerosis in a Portuguese population. Clin Neurol Neurosurg 2013;115:1762-1765.

\footnotetext{
3. Hewitt D, Milner J, Csima A, Pakula A. On Edwards' criterion of seasonality and a non-parametric alternative. Br J Prev Soc Med 1971;25:174-176.

4. Willer CJ, Dyment DA, Sadovnik AD, et al. Timing of birth and risk of multiple sclerosis: population based study. BMJ 2005;330:120.

5. Dobson R, Giovannoni G, Ramagopalan S. The month of birth effect in multiple sclerosis: systematic review, meta-analysis and effect of latitude. J Neurol Neurosurg Psychiatry 2013;84:427-432.
} 